\title{
Competitive Design of Energy Harvesting Communications in Wireless Fading Channels
}

\author{
Jesus Gómez-Vilardebò \\ Centre Tecnològic de Telecomunicacions de Catalunya (CTTC) \\ Castelldefels, Spain \\ jesus.gomez@cttc.es
}

\begin{abstract}
This paper considers the design of online transmission policies for slotted energy harvesting point-to-point communications systems in wireless fading channels. The objective is to minimize the competitive rate gap that is defined as the maximum gap between the optimal rates that can be achieved by the offline, and online transmission policies over all possible energy arrival profiles, and fading states. The paper introduces the competitive rate gap analysis, and solves the particular case of two transmission slots. For two time slots, we show that the optimal competitive rate gap is $0.2075 \mathrm{bits} / \mathrm{s} / \mathrm{Hz}$, and present a transmission policy that achieves it. The new online policy is shown to outperform previously proposed online policies, such as the myopic policy.
\end{abstract}

\section{INTRODUCTION}

Energy harvesting (EH) technology is considered as a major component of future wireless networks. Harvesting energy from the environment extends the lifetime of wireless devices, and provides them untethered mobility, as batteries can be charged without connecting to the power grid infrastructure. However, designing EH communication systems bring its own challenges. For many energy sources, such as solar, vibration or electromagnetic, the characteristics of the EH profile change over time. The time-varying nature of the available energy motivates the need of designing transmission polices that take into account the stochastic nature of the energy arrival process, while optimizing a desired performance criteria.

Previous work addressing the design of transmission polices for $\mathrm{EH}$ devices are typically classified based on the assumptions made on the transmitter's knowledge about the $\mathrm{EH}$ process [1]. In the offline optimization framework the transmitter is assumed to have access to all the future energy packet arrival instants and packet sizes. The optimal offline transmission policy maximizing the throughput for an $\mathrm{EH}$ point-to-point additive white Gaussian noise (AWGN) channel was first studied in [2] and, extended to fading channels in [3]. The offline design serves as a theoretical upper-bound and have also been proven useful in inspiring online policies [4]. However, practical interest in offline polices is limited to scenarios for which the EH process is more or less deterministic, or is random, but can be accurately predicted. For

This work was partially supported by the Catalan Government under grant SGR2014-1567, the Spanish Government under grant PCIN-2013-027 (ECROPS), and TEC2013-44591-P (INTENSYV), and the European project FP7-ICT-2011-8 NEWCOM\# 318306. example, solar based systems and shoe-mounted piezoelectric devices. The online optimization framework, instead, assumes that the future energy arrivals are unknown. If the transmitter has statistical knowledge of the underlying EH process then, the optimization problem is modeled as a Markov decision process, and the optimal policy can be determined through dynamic programming [1]. Most of the works available in the literature about online optimization show performance results that are very close to those achieved by optimal offline policies [5]. However, it is not yet clear how much of these results can be attributed to the particular online policy chosen, or the stochastic model considered for the $\mathrm{EH}$ and fading processes.

In this work, we adopt a competitive (worst-case) analysis framework, for which the statistics of the EH process and the fading channel are not relevant. Our main objective is to characterize the gap between the optimal offline and online policies. Identifying this gap independent of the EH and fading statistics will determine the value of the knowledge about these random processes. If the gap between the optimal offline and online policies is significantly large, more effort should be put into learning the behaviour of the underlying $\mathrm{EH}$ and fading processes [6]. Moreover, the value of the gap will also let us know the value of the offline results as a performance benchmark, a claim commonly used in the literature.

The most related paper to ours is [7], in which the authors, resorting to the competitive analysis framework developed in [8], introduce the competitive analysis for $\mathrm{EH}$ communication systems in terms of the competitive rate ratio as the maximum ratio between the optimal offline rate and online rate. Here, we study, instead, the competitive rate gap. For the competitive rate gap analysis, the competitive ratio analysis framework developed in [8] can not be directly applied. Here, we develop a new framework for the systematic design of algorithm solutions as well as for the establishment of worsecase performance bounds in terms of the competitive rate gap.

The remainder of the paper is organized as follows. The system model is described in Section II. The competitive analysis framework is developed in Section III. The competitive rate gap results for static channels are reviewed in Section IV. In Section $\mathrm{V}$ we address the situation in which a fixed energy is available at the beginning of the transmission, and in Section VI the case of arbitrary time-varying energy arrivals and channels. These results are evaluated numerically in Section VII. Finally, concluding remarks are given in Section VIII. 


\section{System ModeL}

Consider a slotted wireless transmission from a source to a destination over an AWGN fading channel. We assume a block fading model, where the fading coefficients remain constant for the duration of a slot. We denote as $h_{n} \in\left\{0, \mathbb{R}^{+}\right\}$the fading coefficient at time slot $n=1,2, . ., N$, where $N$ is the total number of slots. The source terminal harvests energy from the environment over time. The energy harvested during time slot $n-1$ is only available at the beginning of slot $n$, and is denoted by $E_{n} \in\left\{0, \mathbb{R}^{+}\right\}, n=1,2, \ldots, N$.

We consider the Shannon capacity function to relate the achieved instantaneous rate to the power; that is, if the duration of the communication is $T$, and the transmission power at time $t$ is $p(t)$ then the instantaneous rate is given by $r(p(t))=$ $\log _{2}(1+p(t))$, and the total number of bits transmitted over the period of time $T$ is given by $\int_{0}^{T} r(p(t)) d(t)$. Denote by $U_{n}$ the energy allocated for transmission during time slot $n$. It is well known that, due to the strict concavity of the capacity function, the rate in each slot is maximized by equally distributing the energy $U_{n}$ over the whole slot duration $\frac{T}{N}$. Then, the total number of bits transmitted over slot $n$ is found as follows

$$
D_{n}\left(U_{n}\right)=\frac{T}{N} \log _{2}\left(1+N h_{n} \frac{U_{n}}{T}\right) .
$$

After $N$ time slots, the rate achieved is $R=$ $\frac{1}{T} \sum_{n=1}^{N} D_{n}\left(U_{n}\right)$. Due to the energy causality constraint, the total energy used by the end of slot $n$ cannot be more than the energy harvested by the beginning of timeslot $n$, $n=1,2, \ldots, N$, that is, $U_{m}$ values have to satisfy:

$$
\sum_{m=1}^{n} U_{m} \leq \sum_{m=1}^{n} E_{m}, \forall n \leq N .
$$

Hereafter, without loss of generatively, we consider $T=1$.

\section{Competitive Analysis}

For convenience, let us define $H_{i} \triangleq 2 h_{i} E_{i}, i=1, \ldots, N$, and $\mathbf{H} \triangleq\left\langle H_{1}, h_{1}, H_{2}, h_{2}, \ldots, H_{N}, h_{N}\right\rangle$. Our goal is to study the rate gap between the rate achieved by the optimal offline policy $R_{\mathcal{O}}(\mathbf{H})$, which assumes that the fading channels and energy arrivals $\left(h_{n}, E_{n}\right)$, or equivalently $\left(h_{n}, H_{n}\right)$ for $n=1, \ldots, N$ are known in advance, and the rate $R_{\mathcal{U}}(\mathbf{H})$ achieved by the online policy $\mathcal{U}$ maximized over all possible fading channels and energy harvesting profiles. We want to characterize the minimum value of this maximum rate gap, the competitive rate gap $(g)$, defined as

$$
g=\min _{\mathcal{U}} \max _{\mathbf{H} \in\left\{0, \mathbb{R}^{+}\right\}^{2 N}} R_{\mathcal{O}}(\mathbf{H})-R_{\mathcal{U}}(\mathbf{H}) .
$$

The competitive rate gap here considered resembles the competitive ratio most usually considered in the competitive analysis literature [8]. The competitive ratio for $\mathrm{EH}$ was addressed in [7] for a EH point to point slotted communication over a fading channels, and defined as

$$
r=\min _{\mathcal{U}} \max _{\mathbf{H} \in\left\{0, \mathbb{R}^{+}\right\}^{N}} \frac{R_{\mathcal{O}}(\mathbf{H})}{R_{\mathcal{U}}(\mathbf{H})}
$$

There authors show that, if the power policy is online with respect to both; the $\mathrm{EH}$ input process and the channel fading process, then the competitive ratio is equal to the number of slots $r=N$. The competitive rate gap studied here complements the information provided by the competitive ratio. For the competitive ratio analysis it is sufficient to consider the low power regime. However, rate gaps are maximized in the high power regime. Competitive rate gap optimal online policies might fail in guaranteing a bounded rate gap. Specifically, in [7] authors show that the myopic policy that equally distributes the available energy over the remaining slots, there referred to as repeated equal power allocation (REPA) algorithm is optimal in terms of the competitive ratio. However, as we show here the rate gap obtained by the myopic policy is far from the optimal rate gap.

For the formulation of the completive rate gap problem, we need first to derive explicit expressions for the optimal offline and online rates. An efficient algorithm to compute exactly the optimal offline policy $\mathcal{O}$, and offline rate was presented in [9] refereed to as staircase water filling althorithm, and also in [3] referred to as directional water-filling algorithm. For the particular case of two time-slots $N=2$, the resultant optimal offline policy $\mathcal{O}:\left\langle U_{1}^{(o)}, U_{2}^{(o)}\right\rangle$, can be expressed in closed form as: $U_{1}^{(o)}=\left(E_{1}-\frac{1}{2}\left(\lambda_{1}-\lambda_{2}\right)^{+}\right)^{+}$, and $U_{2}^{(o)}=$ $E_{1}+E_{2}-U_{1}^{(o)}$, where $(x)^{+}=0$ if $x \leq 0$, and $(x)^{+}=x$ otherwise, and where $\lambda_{1}=\frac{1+H_{1}}{2 h_{1}}$, and $\lambda_{2}=\frac{1+H_{2}}{2 h_{2}}$. Then the optimal offline rate $R_{\mathcal{O}}(\mathbf{H})$ is given by

$$
R_{\mathcal{O}}= \begin{cases}R_{\mathrm{A}}, & \text { if } \lambda_{1}>\lambda_{2}, h_{1}\left(\lambda_{1}+\lambda_{2}\right)>1 \\ R_{\mathrm{B}}, & \text { if } \lambda_{1}>\lambda_{2}, h_{1}\left(\lambda_{1}+\lambda_{2}\right)<1, \\ R_{\mathrm{C}}, & \text { if } \lambda_{1}<\lambda_{2},\end{cases}
$$

where

$$
\begin{aligned}
R_{\mathrm{A}} & =\frac{1}{2} \log _{2}\left(h_{2} h_{1}\left(\frac{1+H_{2}}{2 h_{2}}+\frac{1+H_{1}}{2 h_{1}}\right)^{2}\right), \\
R_{\mathrm{B}} & =\frac{1}{2} \log _{2}\left(1+\frac{h_{2}}{h_{1}} H_{1}+H_{2}\right), \\
R_{\mathrm{C}} & =\frac{1}{2} \log _{2}\left(\left(1+H_{1}\right)\left(1+H_{2}\right)\right) .
\end{aligned}
$$

We consider online policies $\mathcal{U}$ that make their decisions based only on the past and current fading coefficients and energy arrivals, and make no assumption about the statistics of the $\mathrm{EH}$ process, namely $\mathcal{U}(\mathbf{H})=\left\langle U_{1}, \ldots, U_{N}\right\rangle$, where the energies spent at time slots $n=1, \ldots, N$ are defined by the functions $U_{n}\left(\mathbf{H}_{n}\right):\left\{0, \mathbb{R}^{+}\right\}^{2 n} \rightarrow\left[0, B_{n}\right]$, where $\mathbf{H}_{n}=$ $\left\langle H_{1}, h_{1}, H_{2}, h_{2}, \ldots, H_{n}, h_{n}\right\rangle$, and $B_{n}$ denotes the amount of energy in the battery at the beginning of time slot $n$. Notice that $U_{n}$ is a fraction of $B_{n}$, which we can write for convenience as $U_{n}\left(\mathbf{H}_{n}\right)=\alpha_{n}\left(\mathbf{H}_{n}\right) B_{n}$ where $0<\alpha_{n}\left(\mathbf{H}_{n}\right) \leq$ 1 and the battery state at time slot $n$, can be computed recursively as $B_{n}=\left(1-\alpha_{n-1}\right) B_{n-1}+E_{n}$ with $B_{n}=0$. It can be argued that any optimal online strategy must spend all the energy harvested by the end of the transmission, and thus we can fix $\alpha_{N}=1$. For the particular case of $N=2$, the 
online policy $\mathcal{U}$ simplifies to $U_{1}\left(\mathbf{H}_{1}\right)=\alpha_{1} E_{1}, U_{2}\left(\mathbf{H}_{2}\right)=$ $\left(1-\alpha_{1}\right) E_{1}+E_{2}$ and the online rate reads

$$
\begin{aligned}
R_{\mathcal{U}}(\mathbf{H})=\frac{1}{2} \log _{2}\left(1+\alpha_{1} H_{1}\right) & \\
& +\frac{1}{2} \log _{2}\left(1+\left(1-\alpha_{1}\right) \frac{h_{2}}{h_{1}} H_{1}+H_{2}\right)
\end{aligned}
$$

We solve (1) by deriving upper- and lower-bounds on $g$ that are tight. The upper-bound is obtained by fixing a particular online policy $\mathcal{U}^{*}$, and solving instead

$$
g_{U}=\max _{\mathbf{H} \in\left\{0, \mathbb{R}^{+}\right\}^{2 N}} R_{\mathcal{O}}(\mathbf{H})-R_{\mathcal{U}^{*}}(\mathbf{H}) .
$$

The lower-bound is obtained by maximizing over a subset $\mathcal{S}$ of all the possible fading, and energy harvesting sequences $\mathbf{H} \in \mathcal{S}$ $\subset\left\{0, \mathbb{R}^{+}\right\}^{2 N}$, and minimizing over all possible policies $\mathcal{U}$,

$$
g_{L}=\operatorname{minmax}_{\mathcal{U}} R_{\mathcal{H} \in \mathcal{S}}(\mathbf{H})-R_{\mathcal{U}}(\mathbf{H}) .
$$

If channel coefficients remain constant over all the transmission slots, but energy arrivals are random, we have used this approach in [10] and [11] to show that the competitive rate gap is upper-bound by $g \leq \log _{2} N$ and lower-bounded by $g_{L}=$ $-\frac{1}{N} \log _{2}\left(\prod_{n=1}^{N} \alpha_{n}^{*}\right)$ where $\alpha_{n}^{*}=1-\sum_{l=2}^{N+1-n} \frac{(l-1)^{l-1}}{(l)^{l}} \alpha_{n+l-1}^{*}$.

The final objective of this work is to extend these results to fading channels. We begin here by considering the case of two time slots. For the general case of $N$ time slots, arbitrary energy arrivals and time-varying channels coefficient, a close form expression for the optimal offline rate needs to define $2^{N}-1$ different regions in a $N$ dimensional space $\left(\lambda_{1}, \ldots, \lambda_{N}\right)$. Solving the competitive rate gap for such case might require a different approach, and is left for future work.

\section{Constant Channel Coefficients}

Let us first review the known results for the situation in which fading coefficients remain constant for the two transmission slots, but the energy harvested amounts change at each time slot. The optimal offline policy and offline rate for any number of slots was found in [12]. For two time slots, the resultant offline rate is given by particularizing $R_{\mathcal{O}}$ in (2), with $h_{1}=h_{2}=h$, obtaining

$$
R_{\mathcal{O}}= \begin{cases}\log _{2}\left(\frac{1+H_{2}}{2}+\frac{1+H_{1}}{2}\right), & \text { if } H_{1}>H_{2}, \\ \left.\frac{1}{2} \log _{2}\left(\left(1+H_{1}\right)^{(1}+H_{2}\right)\right), & \text { if } H_{1}<H_{2} .\end{cases}
$$

Observe that given $H_{1}$ and $H_{2}$ neither the offline, nor the online rates depend on the channel $h$, and thus the maximization in (1) is performed over $\mathbf{H}=\left\langle H_{1}, H_{2}\right\rangle \in\left\{0, \mathbb{R}^{+}\right\}^{2}$. The competitive rate gap was shown in [10] to be

$$
g=\frac{1}{2} \log _{2}\left(\frac{4}{3}\right)=0.2075 \text { bits } / \mathrm{s} / \mathrm{Hz} .
$$

It was also found that the online policy $\mathcal{U}^{*}:\left\langle U_{1}, U_{2}\right\rangle=$ $\left\langle\frac{3}{4} E_{1}, \frac{1}{4} E_{1}+E_{2}\right\rangle$ is competitive rate gap optimal ( $g$ optimal), and that the myopic policy $\mathcal{U}^{m}:\left\langle U_{1}, U_{2}\right\rangle=$ $\left\langle\frac{1}{2} E_{1}, \frac{1}{2} E_{1}+E_{2}\right\rangle$ obtains $g^{\mathrm{m}}=0.5 \mathrm{bits} / \mathrm{s} / \mathrm{Hz}$.

\section{Single Energy Arrival}

Next, we discuss the situation in which energy only arrives at the beginning of the transmission, at slot 1, and no energy arrives after that, namely $E_{2}=H_{2}=0$, while the fading coefficients $h_{1}, h_{2}$ are arbitrarily varying. The offline and online rates are thus a function of $\mathbf{H} \triangleq\left\langle H_{1}, h_{1}, h_{2}\right\rangle \in\left\{0, \mathbb{R}^{+}\right\}^{3}$. In this case, the optimal offline policy is given by the well-known water-filling power allocation for parallel fading channels. For two time slots, the resultant offline rate is given by particularizing $R_{\mathcal{O}}$ in (2) with $H_{2}=0$.

\section{A. Lower-Bound}

To compute a lower-bound on the competitive rate gap, we consider the subset $\mathcal{S}$ of sequences $\mathbf{H}$ that results from limiting $h_{2}$ to belong to $\{0, \infty\}$, and $H_{1}, h_{1} \in\left\{0, \mathbb{R}^{+}\right\}^{2}$, namely

$$
\mathcal{S}=\left\{\mathbf{H}^{(0)}, \mathbf{H}^{(\infty)}: H_{1}, h_{1} \in\left\{0, \mathbb{R}^{+}\right\}^{2}\right\}
$$

where $\mathbf{H}^{(0)}=\left\langle H_{1}, h_{1}, 0\right\rangle$, and $\mathbf{H}^{(\infty)}=\left\langle H_{1}, h_{1}, \infty\right\rangle$. The offline and online rates associated to the input sequences $\mathbf{H}^{(0)}$ and $\mathbf{H}^{(\infty)}$ are given by

$$
\begin{aligned}
R_{\mathcal{O}}\left(\mathbf{H}^{(0)}\right) & =\frac{1}{2} \log _{2}\left(1+H_{1}\right), \\
R_{\mathcal{O}}\left(\mathbf{H}^{(\infty)}\right) & =\lim _{h_{2} \rightarrow \infty} \begin{cases}\frac{1}{2} \log _{2}\left(\frac{h_{2}}{4 h_{1}}\left(1+H_{1}\right)^{2}\right), & \text { if } H_{1} \geq 1, \\
\frac{1}{2} \log _{2}\left(\frac{h_{2}}{h_{1}} H_{1}\right), & \text { if } H_{1}<1,\end{cases} \\
R_{\mathcal{U}}\left(\mathbf{H}^{(0)}\right) & =\frac{1}{2} \log _{2}\left(1+\alpha_{1} H_{1}\right), \\
R_{\mathcal{U}}\left(\mathbf{H}^{(\infty)}\right) & =\lim _{h_{2} \rightarrow \infty} \frac{1}{2} \log _{2}\left(\left(1+\alpha_{1} H_{1}\right)\left(1-\alpha_{1}\right) H_{1} \frac{h_{2}}{h_{1}}\right) .
\end{aligned}
$$

Define $G(\mathbf{H}) \triangleq R_{\mathcal{O}}(\mathbf{H})-R_{\mathcal{U}}(\mathbf{H})$. The lower-bound on the competitive rate gap can be computed by solving

$$
g_{L}=\min _{\alpha_{1}(\mathbf{H}) \mathbf{H} \in \mathcal{S}} \max _{\mathbf{H}} G(\mathbf{H}) .
$$

If $H_{1}>1, G\left(\mathbf{H}^{(0)}\right)$ monotonically decreases with $\alpha_{1}$, whereas $G\left(\mathbf{H}^{(\infty)}\right)$ has a minimum at $\alpha_{1}=\bar{\alpha}_{1} \triangleq \frac{H_{1}-1}{2 H_{1}}$, monotonically decreases for $0 \leq \alpha_{1}<\bar{\alpha}_{1}$, and monotonically increases for $\bar{\alpha}_{1}<\alpha_{1} \leq 1$. The equality $G\left(\mathbf{H}^{(\infty)}\right)=$ $G\left(\mathbf{H}^{(0)}\right)$ is obtained at $\alpha_{1}=\alpha_{1 \mid H_{1}>1}^{*} \triangleq \frac{3 H_{1}-1}{4 H_{1}}$. Given that $\alpha_{1 \mid H_{1}>1}^{*}>\bar{\alpha}_{1}$, the minimum in (6) for any $H_{1}$ and $h_{1}$ is found at $\alpha_{1}=\alpha_{1 \mid H_{1}>1}^{*}$, as $\left.G\left(\mathbf{H}^{(0)}\right)\right|_{\alpha_{1}=\alpha_{1 \mid H_{1}>1}^{*}}=\frac{1}{2} \log _{2}\left(\frac{4}{3}\right)$.

If $H_{1}<1, G\left(\mathbf{H}^{(0)}\right)$ monotonically decreases with $\alpha_{1}$, whereas $G\left(\mathbf{H}^{(\infty)}\right)$ monotonically decreases for $0 \leq \alpha_{1}<\bar{\alpha}_{1}$ and increases for $\bar{\alpha}_{1}<\alpha_{1} \leq 1$. For $G\left(\mathbf{H}^{(\infty)}\right)=G\left(\mathbf{H}^{(0)}\right)$ now we need $\alpha_{1}=\alpha_{1 \mid H_{1}<1}^{*} \triangleq \frac{H_{1}}{1+H_{1}}$. Given that $\frac{H_{1}}{1+H_{1}}>$ $\frac{H_{1}-1}{2 H_{1}}$, the minimum rate gap for any $H_{1}$ and $h_{1}$ is found at $\alpha_{1}=\alpha_{1 \mid H_{1}<1}^{*}$, as

$$
\begin{aligned}
\left.G\left(\mathbf{H}^{(0)}\right)\right|_{\alpha_{1}=\alpha_{1 \mid H_{1}<1}^{*}} & =-\frac{1}{2} \log _{2}\left(1-\frac{H_{1}}{\left(1+H_{1}\right)^{2}}\right) \\
& \leq \frac{1}{2} \log _{2}\left(\frac{4}{3}\right)
\end{aligned}
$$


Finally, since $\left.G\left(\mathbf{H}^{(0)}\right)\right|_{\alpha_{1}=\alpha_{1 \mid H_{1}<1}^{*}} \leq \frac{1}{2} \log _{2}\left(\frac{4}{3}\right)$, the competitive rate gap in (6) is given by $g_{L}=\frac{1}{2} \log _{2}\left(\frac{4}{3}\right) \mathrm{bits} / \mathrm{s} / \mathrm{Hz}$, and is achieved with equality at $H_{1}=1$. Notice that this lower-bound coincides with the competitive rate gap for the case of constant channel gains, and random energy arrivals.

\section{B. Upper-Bound}

The derivation of the lower-bound suggest the online policy $\mathcal{U}^{*}:\left\langle U_{1}, U_{2}\right\rangle=E_{1}\left\langle\alpha_{1}^{*}, 1-\alpha_{1}^{*}\right\rangle$, where

$$
\alpha_{1}^{*}\left(H_{1}\right)= \begin{cases}\frac{H_{1}}{1+H_{1}}, & \text { if } H_{1}<1, \\ \frac{3 H_{1}-1}{4 H_{1}}, & \text { if } H_{1}>1 .\end{cases}
$$

We obtain an upper-bound on the competitive gap by fixing $\mathcal{U}^{*}$, and solving (4), for the 3 regions in which the offline rate (2) is defined:

The offline rate is given by $R_{\mathcal{O}}=R_{\mathrm{A}}(\mathbf{H})$, for any $h_{2}$ satisfying $\check{h}_{2} \leq h_{2}$, and $h_{2} \leq \hat{h}_{2}$ if $1>H_{1}$ where $\check{h}_{2} \triangleq \frac{h_{1}}{1+H_{1}}$, and $\hat{h}_{2} \triangleq \frac{h_{1}}{1-H_{1}}$. The rate gap $G_{\mathrm{A}}^{*}(\mathbf{H})=R_{\mathrm{A}}(\mathbf{H})-R_{\mathcal{U}^{*}}(\mathbf{H})$ as a function of $h_{2}$ has a minimum at $h_{2}=\bar{h}_{2} \triangleq \frac{h_{1}}{1-\left(1-2 \alpha_{1}^{*}\right) H_{1}}$, decreases monotonically for $h_{2}<\bar{h}_{2}$, and increases monotonically for $h_{2}>\bar{h}_{2}$. Observe that $\check{h}_{2} \leq \bar{h}_{2} \leq \hat{h}_{2}$. Consequently, $G_{\mathrm{A}}^{*}(\mathbf{H})$ is maximum either at $h_{2}=\check{h}_{2}$, at $h_{2} \rightarrow \infty$ if $1<H_{1}$, or at $h_{2}=\hat{h}_{2}$ if $1>H_{1}$. We bound the rate gap in each of these situations:

At $h_{2}=\breve{h}_{2}$ if $1>H_{1}$, or $1<H_{1}$, we have

$$
\begin{aligned}
G_{\mathrm{A} \mid 1>H_{1}}^{*}\left(\left\langle H_{1}, h_{1}, \check{h}_{2}\right\rangle\right) & =-\frac{1}{2} \log _{2}\left(1-\frac{\left(H_{1}\right)^{2}}{\left(1+H_{1}\right)^{4}}\right), \\
& \leq \frac{1}{2} \log _{2}\left(\frac{16}{15}\right) . \\
G_{\mathrm{A} \mid 1<H_{1}}^{*}\left(\left\langle H_{1}, h_{1}, \check{h}_{2}\right\rangle\right) & =\frac{1}{2} \log _{2}\left(\frac{16}{15}\right) .
\end{aligned}
$$

At $h_{2} \rightarrow \infty$ if $1<H_{1}, G_{\mathrm{A}}^{*}\left(\left\langle H_{1}, h_{1}, \infty\right\rangle\right)=\frac{1}{2} \log _{2}\left(\frac{4}{3}\right)$.

At $h_{2}=\hat{h}_{2}$ and $1>H_{1}$, we have

$$
\begin{aligned}
G_{\mathrm{A}}^{*}\left(\left\langle H_{1}, h_{1}, \hat{h}_{2}\right\rangle\right) & =-\frac{1}{2} \log _{2}\left(1-\frac{\left(H_{1}\right)^{4}}{\left(1+H_{1}\right)^{2}}\right), \\
& \leq \frac{1}{2} \log _{2}\left(\frac{4}{3}\right) .
\end{aligned}
$$

Consequently $G_{\mathrm{A}}^{*}(\mathbf{H}) \leq \frac{1}{2} \log _{2}\left(\frac{4}{3}\right)$.

If the offline rate is given by $R_{\mathcal{O}}=R_{\mathrm{B}}(\mathbf{H})$, the rate gap $G_{\mathrm{B}}^{*}(\mathbf{H})=R_{\mathrm{B}}(\mathbf{H})-R_{\mathcal{U}^{*}}(\mathbf{H})$ increases monotonically with $h_{2}$. The conditions for $R_{\mathcal{O}}=R_{\mathrm{B}}(\mathbf{H})$ require $1 \geq H_{1}$, but allow $h_{2} \rightarrow \infty$, and thus

$$
\begin{aligned}
G_{\mathrm{B}}^{*}\left(\left\langle H_{1}, h_{1}, \infty\right\rangle\right) & =-\frac{1}{2} \log _{2}\left(1-\frac{H}{\left(1+H_{1}\right)^{2}}\right), \\
& \leq \frac{1}{2} \log _{2}\left(\frac{4}{3}\right) .
\end{aligned}
$$

Finally, if the offline rate is given by $R_{\mathcal{O}}=R_{\mathrm{C}}(\mathbf{H})$, the rate gap $G_{\mathrm{C}}^{*}(\mathbf{H})=R_{\mathrm{C}}(\mathbf{H})-R_{\mathcal{U}^{*}}(\mathbf{H})$ monotonically decreases with $h_{2}$. The conditions for $R_{\mathcal{O}}=R_{\mathrm{C}}(\mathbf{H})$ allow $h_{2}=0$, and the rate gap depending if $1>H_{1}$, or $1<H_{1}$ is bounded by

$$
\begin{aligned}
G_{\mathrm{C} \mid 1>H_{1}}^{*}\left(\left\langle H_{1}, h_{1}, 0\right\rangle\right) & =-\frac{1}{2} \log _{2}\left(1-\frac{H_{1}}{\left(1+H_{1}\right)^{2}}\right), \\
& \leq \frac{1}{2} \log _{2}\left(\frac{4}{3}\right) . \\
G_{\mathrm{C} \mid 1<H_{1}}^{*}\left(\left\langle H_{1}, h_{1}, 0\right\rangle\right) & =\frac{1}{2} \log _{2}\left(\frac{4}{3}\right) .
\end{aligned}
$$

and thus $G_{\mathrm{C}}^{*}(\mathbf{H}) \leq \frac{1}{2} \log _{2}\left(\frac{4}{3}\right)$. The competitive rate gap is then upper-bounded by $g_{u}^{*}=\frac{1}{2} \log _{2}\left(\frac{4}{3}\right)$.

Given that the lower- and the upper-bounds coincide, we conclude that the competitive rate gap is also $g=\frac{1}{2} \log _{2}\left(\frac{4}{3}\right)$ $\mathrm{bits} / \mathrm{s} / \mathrm{Hz}$. Moreover, we have that the online policy (7) is goptimal. Using similar arguments it can be show that for the myopic policy $\mathcal{U}^{\mathrm{m}}$, the rate gaps $G_{\mathrm{X}}^{(\mathrm{m})}=R_{\mathrm{X}}-R_{\mathcal{U}^{\mathrm{m}}}$, with $\mathrm{X} \in$ $\{$ A,B,C $\}$ are upper-bounded by $G_{\mathrm{A}}^{(\mathrm{m})} \leq \frac{1}{2} \log _{2}\left(\frac{4}{3}\right), G_{\mathrm{B}}^{(\mathrm{m})} \leq=$ $\frac{1}{2}$, and $G_{\mathrm{C}}^{(\mathrm{m})} \leq \frac{1}{2}$, and thus the competitive rate gap for the myopic policy is $g^{(\mathrm{m})}=0.5 \mathrm{bits} / \mathrm{s} / \mathrm{Hz}$.

\section{ARbitrary ENERgy ArRivals AND Channels}

Finally, we obtain the competitive rate gap for a scenario with random energy arrivals $E_{1}, E_{2}$, and arbitrarily varying fading coefficients $h_{1}$, and $h_{2}$ at each slot. As a lower-bound on the competitive gap, we can invoke any of the lower-bounds presented in previous sections as they were obtained with subset of sequences $\mathbf{H}=\left\langle H_{1}, h_{1}, H_{2}, h_{2}\right\rangle \in\left\{0, \mathbb{R}^{+}\right\}^{4}$. Here, we thus focus on the derivation of a tight upper-bound. To that end, we fix the online policy (7), and solve (4), separately, for each of the offline rate expressions in (2).

The offline rate is given by $R_{\mathcal{O}}=R_{\mathrm{A}}(\mathbf{H})$ if $H_{2}$ satisfies $\hat{H}_{2}>H_{2}>\check{H}_{2}$ where $\hat{H}_{2} \triangleq \frac{h_{2}}{h_{1}}\left(1+H_{1}\right)-1$, and $\check{H}_{2} \triangleq$ $\frac{h_{2}}{h_{1}}\left(1-H_{1}\right)-1$. The rate gap $G_{\mathrm{A}}^{*}(\mathbf{H})$ as a function of $H_{2}$, has a minimum at $\bar{H}_{2}=\frac{h_{2}}{h_{1}}\left(1+\left(2 \alpha_{1}-1\right) H_{1}\right)-1$, monotonically decreases for $H_{2}<\bar{H}_{2}$, and increases otherwise. Consequently, the rate gap is maximized either at $H_{2}=\hat{H}_{2}$, at $H_{2}=\check{H}_{2}$ if $\check{H}_{2}>0$, or at $H_{2}=0$ if $\check{H}_{2}<0$ and $\bar{H}_{2}>0$. Evaluating each of these cases, we have:

If $H_{2}=0$, we known from previous section that $G_{\mathrm{A}}\left(\left\langle H_{1}, h_{1}, 0, h_{2}\right\rangle\right) \leq \frac{1}{2} \log _{2}\left(\frac{4}{3}\right)$.

If $H_{2}=\hat{H}_{2}$ and $1>H_{1}$, or $1<H_{1}$, we have

$$
\begin{aligned}
G_{\mathrm{A} \mid 1>H_{1}}^{*}\left(\left\langle H_{1}, h_{1}, \hat{H}_{2}, h_{2}\right\rangle\right) & =-\frac{1}{2} \log _{2}\left(1-\frac{H_{1}^{2}}{\left(1+H_{1}\right)^{4}}\right), \\
& \leq \frac{1}{2} \log _{2}\left(\frac{16}{15}\right) . \\
G_{\mathrm{A} \mid 1<H_{1}}^{*}\left(\left\langle H_{1}, h_{1}, \hat{H}_{2}, h_{2}\right\rangle\right) & =\frac{1}{2} \log _{2}\left(\frac{16}{15}\right) .
\end{aligned}
$$

If $H_{2}=\check{H}_{2}$, and $\check{H}_{2}>0$ a necessary condition is $1>H_{1}$. 
Thus, the rate gap is bounded by

$$
\begin{aligned}
G_{\mathrm{A}}^{*}\left(\left\langle H_{1}, h_{1}, \check{H}_{2}, h_{2}\right\rangle\right) & =-\frac{1}{2} \log _{2}\left(1-\frac{\left(H_{1}\right)^{4}}{\left(1+H_{1}\right)^{2}}\right), \\
& \leq \frac{1}{2} \log _{2}\left(\frac{4}{3}\right) .
\end{aligned}
$$

We thus conclude that $G_{\mathrm{A}}^{*}(\mathbf{H}) \leq \frac{1}{2} \log _{2}\left(\frac{4}{3}\right)$.

If the offline rate is given by $R_{\mathcal{O}}=R_{\mathrm{B}}(\mathbf{H})$, the rate gap $G_{\mathrm{B}}^{*}(\mathbf{H})$ decreases monotonically with $H_{2}$. By letting $H_{2} \rightarrow 0$, we are in the scenario considered in the previous section, and we can bound $G_{\mathrm{B}}^{*}\left(\left\langle H_{1}, h_{1}, 0, h_{2}\right\rangle\right) \leq \frac{1}{2} \log _{2}\left(\frac{4}{3}\right)$.

Finally, if the offline rate is given by $R_{\mathcal{O}}=R_{\mathrm{C}}(\mathbf{H})$, the rate gap, increases monotonically with $H_{2}$. By letting $H_{2} \rightarrow \infty$, if $H_{1} \leq 1$, we obtain

$$
\begin{aligned}
G_{\mathrm{C} \mid H_{1} \leq 1}^{*}\left(\left\langle H_{1}, h_{1}, \infty, h_{2}\right\rangle\right) & =-\frac{1}{2} \log _{2}\left(1-\frac{H_{1}}{\left(1+H_{1}\right)^{2}}\right), \\
& \leq \frac{1}{2} \log _{2}\left(\frac{4}{3}\right) .
\end{aligned}
$$

else if $H_{1}>1$, then $G_{\mathrm{C} \mid H_{1}>1}^{*}\left(\left\langle H_{1}, h_{1}, \infty, h_{2}\right\rangle\right)=\frac{1}{2} \log _{2}\left(\frac{4}{3}\right)$. Thus, we conclude that the competitive rate gap is given by $g=\frac{1}{2} \log _{2}\left(\frac{4}{3}\right)$.

\section{NumericAl RESUlts}

In this section, we validate numerically the competitive rate gap obtained, and illustrate the competitive rate gap for several online policies. For the case of $N=2$, we can obtain numerically the competitive rate gap, as well as, the optimal online policy for any $h_{1}$, and $H_{1}$, by solving

$$
g\left(H_{1}, h_{1}\right)=\min _{\alpha\left(H_{1}, h_{1}\right)} \max _{H_{2}, h_{2}} R_{\mathcal{O}}-R_{\mathcal{U}}
$$

We solve (8), by exhaustive search. We limit $H_{2}$ and $h_{2}$ to belong to the finite set $\{0,0.01, \ldots, 100\}$, and $\alpha \in$ $\{0,0.01, \ldots, 1\}$. The resultant competitive rate gap as a function of $H_{1}=2 h_{1} E_{1}$ is depicted in Fig. 8, together with the rate gaps obtained by the optimal online policy presented here, the optimal online policy for static channels but random energy arrivals, and the myopic policy. Observe that the goptimal strategy proposed in (7) reaches the competitive rate gap already at $H_{1}=1$, but never surpasses this value. The g-optimal strategy for static channels instead converges from above to the competitive rate gap as $H_{1}$ increase.

\section{CONCLUSIONS}

We studied the competitive rate gap for $\mathrm{EH}$ communication systems in fading wireless channels. For two-time slots, we found the competitive rate gap, which is defined as the maximum difference between the rate obtained with an offline power policy and an online power policy. We showed that the competitive rate gap is equal to $0.2075 \mathrm{bits} / \mathrm{s} / \mathrm{Hz}$. Interestingly, the competitive rate gap remains the same, if we consider static channels but random energy arrivals, or if we only consider the fading process. We proposed an online strategy that archives the optimal competitive rate gap, and validate

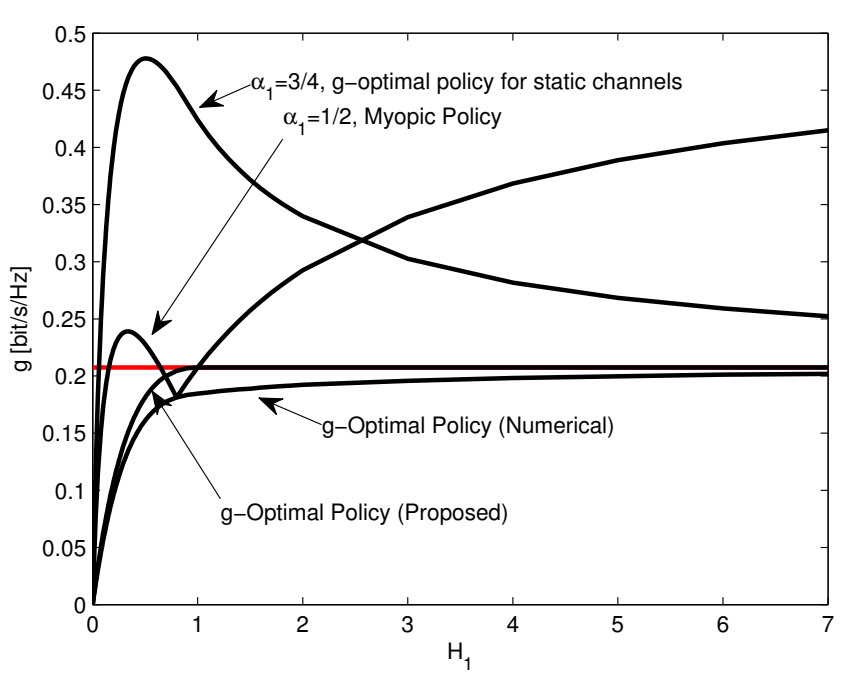

Fig. 1: Competitive rate gap as a function of $H_{1}$, for $N=2$.

the results numerically. Future immediate work should address the problem of obtaining the competitive rate gap for the case of $N$ slots. The extension of this analysis to multi-terminal communications, such as the multiple access, the broadcast, the relay and the interference channel are also interesting.

\section{REFERENCES}

[1] D. Gunduz, K. Stamatiou, N. Michelusi, and M. Zorzi, "Designing intelligent energy harvesting communication systems," Communications Magazine, IEEE, vol. 52, no. 1, pp. 210-216, January 2014.

[2] J. Yang and S. Ulukus, "Optimal packet scheduling in an energy harvesting communication system," Communications, IEEE Transactions on, vol. 60, no. 1, pp. 220-230, January 2012.

[3] O. Ozel, K. Tutuncuoglu, J. Yang, S. Ulukus, and A. Yener, "Transmission with energy harvesting nodes in fading wireless channels: Optimal policies," Selected Areas in Communications, IEEE Journal on, vol. 29, no. 8, pp. 1732-1743, September 2011.

[4] _ - "Transmission with energy harvesting nodes in fading wireless channels: Optimal policies," Selected Areas in Communications, IEEE Journal on, vol. 29, no. 8, pp. 1732-1743, September 2011.

[5] M. Kashef and A. Ephremides, "Optimal packet scheduling for energy harvesting sources on time varying wireless channels," Communications and Networks, Journal of, vol. 14, no. 2, pp. 121-129, April 2012.

[6] P. Blasco, D. Gunduz, and M. Dohler, "A learning theoretic approach to energy harvesting communication system optimization," Wireless Communications, IEEE Transactions on, vol. 12, no. 4, pp. 1872-1882, April 2013.

[7] R. Vaze, R. Garg, and N. Pathak, "Dynamic power allocation for maximizing throughput in energy-harvesting communication system," pp. 1-1, 2013.

[8] A. Borodin and R. El-Yaniv, Online Computation and Competitive Analysis. New York, NY, USA: Cambridge University Press, 1998.

[9] C. K. Ho and R. Zhang, "Optimal energy allocation for wireless communications powered by energy harvesters," in Information Theory Proceedings (ISIT), 2010 IEEE International Symposium on, June 2010.

[10] J. Gomez-Vilardebo and D. Gunduz, "Competitive analysis of energy harvesting wireless communication systems," in European Wireless (EW) Conference, May 2014.

[11] J. Gomez-Vilardebo, "Competitive design of power allocation strategies for energy harvesting wireless communication systems," in 2nd IEEE Global Conference on Signal and Information Processing, Dec. 2014.

[12] J. Yang and S. Ulukus, "Optimal packet scheduling in an energy harvesting communication system," Communications, IEEE Transactions on, vol. 60, no. 1, pp. 220-230, January 2012. 